Document downloaded from:

http://hdl.handle.net/10251/148187

This paper must be cited as:

Company Rossi, R.; Egorova, VN.; Jódar Sánchez, LA.; Soleymani, F. (2019). A stable local radial basis function method for option pricing problem under the Bates model. Numerical Methods for Partial Differential Equations. 35(3):1035-1055.

https://doi.org/10.1002/num.22337

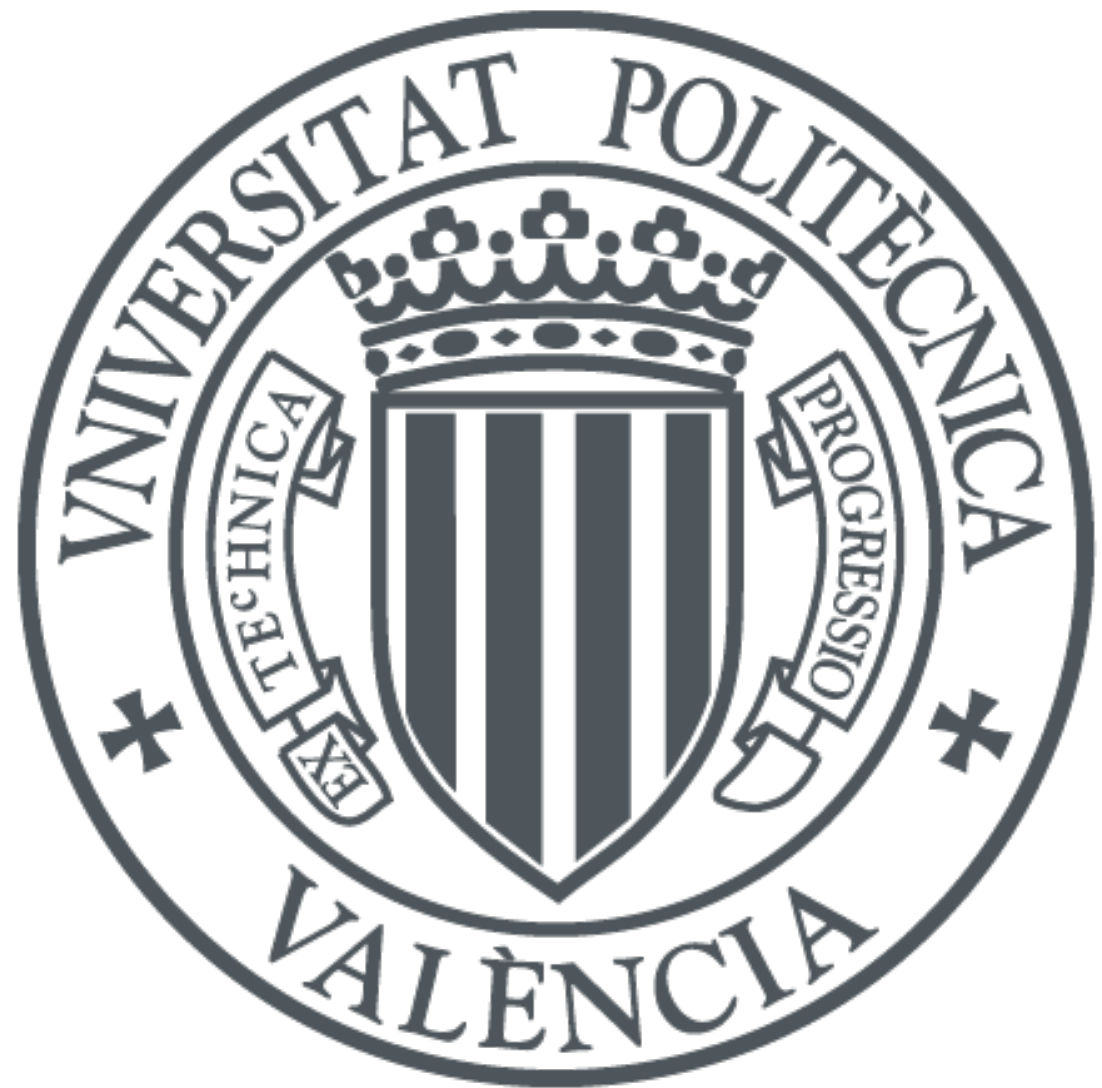

The final publication is available at

https://doi.org/10.1002/num.22337

Copyright John Wiley \& Sons

Additional Information 


\title{
A stable local RBF method for option pricing problem under the Bates model
}

\author{
Rafael Company ${ }^{a, 1}$, Vera N. Egorova ${ }^{b, 2}$, Lucas Jódar ${ }^{a, 3}$, Fazlollah Soleymani ${ }^{c, 4}$ \\ ${ }^{a}$ Instituto Universitario de Matemática Multidisciplinar, Universitat Politècnica de València, Camino de Vera s/n, 46011, València, Spain \\ ${ }^{b}$ BCAM - Basque Center for Applied Mathematics, Alameda de Mazarredo 14, 48009 Bilbao, Basque Country, Spain \\ ${ }^{c}$ Department of Mathematics, Institute for Advanced Studies in Basic Sciences (IASBS), Zanjan 45137-66731, Iran
}

\begin{abstract}
We propose a local mesh-free method for the Bates-Scott option pricing model, a 2D partial integro-differential equation (PIDE) arising in computational finance. A Wendland radial basis function (RBF) approach is used for the discretization of the spatial variables along with a linear interpolation technique for the integral operator. The resulting set of ordinary differential equations (ODEs) is tackled via a time integration method. A potential advantage of using RBFs is the small number of discrete equations that need to be solved. Computational experiments are presented to illustrate the performance of the contributed approach.
\end{abstract}

Keywords: Option pricing; Bates-Scott model; stochastic volatility; radial basis functions; Wendland function 2010 MSC: 91G60; 91B25; 65M22

\section{Introduction}

Several models for option pricing yield partial differential equations (PDEs) in two independent variables, viz, the time and the price of the underlying asset, [1, chapter 2]. Only for a few cases, e.g., the Black-Scholes equation for European call and put options, such PDEs have exact closed-form solutions. Therefore, in general, they must be solved approximately. This can be done using several well-known methods such as finite difference (FD) [35, chapter 3], adaptive FD [37], finite element (FE) [4] and radial basis functions (RBFs) [2] methods, whereas each of them has some domain of practicality.

Jump-diffusion models (see, e.g., [26, 28]) have become an important modeling tool in the pricing of financial derivatives. In the seminal paper [30] in 1976, Merton proposed the addition of jumps into the Black-Scholes model. Contrary to models with continuous paths, jump-diffusion models allow large sudden changes in the price of the underlying asset. Jumps can appear at random times. Because of this, a number of alternative models (in contrast to the Black-Scholes model) have been proposed, see for more [14]. These jump-diffusion models for pricing American options are governed by a parabolic integro-differential variational inequality which can be formulated as a free boundary problem. Mostly, these models contain a differential term and a non-local integral term.

To illustrate further, it is known that for pricing options that have particularly short maturities it is necessary to modify the Black-Scholes equation, see e.g., [8]. Melino and Turnbull showed in [29] that the assumption of stochastic volatility leads to a distribution of the underlying which is closer to empirical observations than the log-normal distribution.

Bates and Scott (see $[10,33])$ proposed simultaneously models incorporating both jumps in the underlying asset and stochastic volatility combining Merton model and Heston's stochastic volatility model [24].

Several techniques have been used to solve (different forms of) the jump-diffusion model such as FD and (RBF) meshfree techniques. The FD method with second order approximations was proposed in [15] for solving

\footnotetext{
${ }^{1}$ Corresponding author. Email address: rcompany@imm.upv.es

${ }^{2}$ Email address: egorova.vn@gmail.com

${ }^{3}$ Email address: ljodar@imm.upv.es

${ }^{4}$ Email address: fazlollah.soleymani@gmail.com \& soleymani@iasbs.ac.ir
} 
simpler versions of the Bates models, i.e., 1D jump diffusion and Levy processes. A FD approach after imposing a transformation to remove the cross derivative term was studied in [18] for the Heston model.

The RBF meshfree technique was demonstrated by solving the PIDE in the one-dimensional Merton case [11] for the American vanilla put and the European vanilla call/put options on dividend-paying stocks. The RBF method in the weak form for the one-dimensional form of the Bates model, i.e., without stochastic volatility has recently been discussed in [2].

There are even fewer works, for problems which additionally include jumps in the underlying's process, and require the solution of a PIDE in two or more spatial dimensions. We mention [37] (and the references cited therein) that propose an implicit-explicit time discretization in combination with a standard second-order FD discretization in space. In addition, high-order FD schemes (fourth order in space) have been proposed for solving PDEs arising from stochastic volatility models [17]. The non-locality of the jump terms in these models leads to matrices with full matrix blocks. Furthermore, a combination of boundary conditions and a mixed second derivative term in the PIDE due to the correlation of the volatility and the stock process exhibit difficulties.

A numerical method for pricing American options under the Bates model has been proposed by Toivanen in [36], where a FD scheme coupled with a componentwise splitting technique is employed. However, according to the results presented therein, this numerical scheme is not particularly fast. To discuss more, the American problem is solved as a linear complementarity problem, and then a time-consuming fixed-point iteration procedure must be performed at each time step. Moreover, the componentwise splitting technique employed in [36] requires a special treatment of the mixed second-order derivatives, which may cause some loss of accuracy, and imposes severe restrictions on the choice of the grid step sizes when a non-uniform mesh is employed.

Meshless methods (also known as meshfree methods) have progressed remarkably in the last decades and some works have been devoted to their classification. The classification can be done based on different criteria, for example, formulation procedure, shape parameter or the domain representation [20].

Meshless methods are specifically useful in the context of computational finance, where the mixed derivative terms are dealt with comparative ease. Handling of mixed derivatives by the classical FE [4] or FD schemes methods is quite complicated and hence meshless methods are the natural choice in such cases. Another merit related to the meshfree schemes is their flexibility over the computational domain and the distribution of the supporting nodes in this domain.

In this work, the motivation of choosing this approach is that along with the above-mentioned merits, the local meshfree methods yields to sparse interpolation matrices and are less sensitive to the choice of the shape parameter [22]. In addition, it avoids mesh-generation, which is the major problem in the FD, FE and spectral methods [31]. Furthermore, one of the state-of-the-art methods for pricing European options under the Bates model is based on Fourier transforms, which is fast and competitive. However, the proposed method in this work aims also at pricing American options.

The paper is organized as follows. In Section 2, we consider a model with stochastic volatility and jumps in returns described by Bates in [9]. Under this model, the behavior of the asset value $S$ and its variance $v$ is described by a system of coupled stochastic differential equations. Discussion on how to model the option for the American type is also furnished therein. Section 3 is devoted to the main contribution of this work whereas a local RBF method is presented for the numerical solution of Bates model. As a matter of fact, the advent of the local meshfree method (in this context) is new.

Moreover, by coupling of the local meshfree method with accurate time integrators needs to be explored for the purpose of completeness. The discretization of the improper integral operator involved in the Bates model is given in Section 4 . Thence, the discretization in time which is a key element in solving the Bates model via the semidiscretization technique is brought forward in Section 5 based on two simple yet effective time-stepping solvers. In the current paper, we concentrate on coupling of a range of time integrators with the local meshfree method. The numerical results obtained are presented and discussed in Section 6. Finally in Section 7, some conclusions are drawn. 


\section{Option pricing model}

The Bates model [9] uses the Black-Scholes equation but also introduces a jump-diffusion term as follows:

$$
\left\{\begin{array}{l}
d S(t)=(r-q-\lambda \xi) S(t) d t+\sqrt{(\nu)} S(t) d W_{1}+(\eta-1) S(t) d Z(t), \\
d \nu(t)=\kappa(\theta-\nu(t)) d t+\sigma \sqrt{(\nu)} d W_{2}, \\
d W_{1} d W_{2}=\rho d t
\end{array}\right.
$$

wherein $W_{1}$ and $W_{2}$ are standard Brownian motions, $Z(t)$ is the Poisson process. The Poisson process is a special case of a so-called counting process. Here $r$ is the risk free rate, $q$ is the dividend yield, $\xi$ is the mean jump, $\eta$ is the jump size, i.e., a random variable measuring the jump amplitude and is assumed to be log-normally distributed in $[0,+\infty), \lambda$ is the intensity of the Poisson process, $\kappa$ is the rate of reversion of the variance $\nu$, while $\theta$ and $\sigma$ are the mean level and volatility constant, respectively. It is worth noticing that if in (1) we set $\eta=1$ (i.e., we exclude the jumps), the vector stochastic differential equation (1) is reduced to the popular Heston model with stochastic volatility.

Actually, the Heston stochastic volatility model [24] and the Merton jump-diffusion model [30] are combined to describe the behavior of the underlying asset $S$ and its variance $\nu$. This results in the following PIDE for the unknown option price $U(S, \nu, \tau)$ as comes next:

$$
\begin{gathered}
\frac{\partial U}{\partial \tau}=\frac{1}{2} \nu S^{2} \frac{\partial^{2} U}{\partial S^{2}}+\rho \sigma \nu S \frac{\partial^{2} U}{\partial S \partial \nu}+\frac{1}{2} \sigma^{2} \nu \frac{\partial^{2} U}{\partial \nu^{2}}+(r-q-\lambda \xi) S \frac{\partial U}{\partial S} \\
+\kappa(\theta-\nu) \frac{\partial U}{\partial \nu}-(r+\lambda) U+\lambda \int_{0}^{\infty} U(S \eta, \nu, \tau) f(\eta) d \eta
\end{gathered}
$$

where $\tau=T-t$ (is the time to expiry), $\xi=\mathbb{E}[\eta-1]$ and the density function $f(\eta)$ is given by [37, p. 2517]:

$$
f(\eta)=\frac{1}{\sqrt{2 \pi} \hat{\sigma} \eta} \exp \left[-\frac{\left(\ln (\eta)-\left(\gamma-\frac{1}{2} \hat{\sigma}^{2}\right)\right)^{2}}{2 \hat{\sigma}^{2}}\right] .
$$

Here the mean $\gamma$ and the standard deviation $\hat{\sigma}$ are constants. Furthermore, we have

$$
\xi=\exp (\gamma)-1
$$

The initial condition for the put and call options is defined by the payoff function, respectively,

$$
U(S, \nu, 0)=p_{0}(S)=\max \{E-S, 0\}
$$

and

$$
U(S, \nu, 0)=p_{0}(S)=\max \{S-E, 0\}
$$

where $E$ is the strike price.

The solution $U(S, \nu, \tau)$ must satisfy suitable boundary conditions which can be derived from the PIDE (2) and the initial condition (5) or (6); and are usually taken into account when solving (2) by a numerical approximation. In particular, for a call option we have

$$
\begin{aligned}
& U(S, \nu, \tau) \approx 0, \quad S \rightarrow 0, \\
& U(S, \nu, \tau) \approx S e^{-q \tau}-E e^{-r \tau}, \quad S \rightarrow+\infty, \\
& \frac{\partial U(S, \nu, \tau)}{\partial \nu} \approx 0, \quad \nu \rightarrow+\infty .
\end{aligned}
$$

It is remarked that the boundary conditions have been given only for $S \rightarrow 0, S \rightarrow+\infty$ and $\nu \rightarrow+\infty$. In fact, for $\nu \rightarrow 0$ the PIDE (2) is singular ([7, p. 1307]) and thus it is not exactly clear, which condition (if any) the 
function $U(S, \nu, \tau)$ has to satisfy. Therefore in the following, using a common approach, we will not prescribe any boundary condition for $\nu \rightarrow 0$. In a sense, we let the linear complementarity problem itself impose the boundary conditions for $\nu \rightarrow 0$ (see, e.g., [12]). Noting that at the boundary condition in $\nu=0, U$ converges to the price in the corresponding Merton model.

The question about the existence of a solution strongly depends on which space we are seeking the solution to be in. We need the integrability of the solution, i.e. their norms must be finite, where the norm is derived from the PIDE. Accordingly, it is assumed that a viscosity solution exist for the PIDE problem (2).

To tackle the American-type option pricing based on Bates model, a similar model can be obtained by formulating a linear complementarity problem (LCP) with a same operator. However, a popular alternative approximation is the penalty method. A nonlinear term as a penalty term according to the idea of [21] is imposed on (2) in order to make the original option pricing PIDE with free boundaries into a nonlinear PIDE for American options as follows:

$$
\begin{aligned}
\frac{\partial U}{\partial \tau}=\frac{1}{2} \nu S^{2} \frac{\partial^{2} U}{\partial S^{2}}+\rho \sigma \nu S \frac{\partial^{2} U}{\partial S \partial \nu}+\frac{1}{2} \sigma^{2} \nu \frac{\partial^{2} U}{\partial \nu^{2}}+(r-q-\lambda \xi) S \frac{\partial U}{\partial S} \\
+\kappa(\theta-\nu) \frac{\partial U}{\partial \nu}-(r+\lambda) U+\lambda \int_{0}^{\infty} U(S \eta, \nu, \tau) f(\eta) d \eta+F(U)
\end{aligned}
$$

In fact, the idea is quite straightforward since in the limit as the positive penalty parameter $\varrho, \varrho \rightarrow \infty$, the solution satisfies $U \geq U^{*}-\epsilon$, for $\epsilon>0, \epsilon \ll 1$, (here $U^{*}$ denotes the payoff). In fact, the solution of the penalty problem is an approximate solution to the discrete linear complementarity problem. It is recalled that convergence of monotone schemes for jump diffusion models is discussed in [14, 15].

One of the ways for introducing the penalty term is [13]:

$$
F(U)=\frac{2 \varrho[(U(S, \nu, 0)-U(S, \nu, \tau))]}{1+\exp \left(-\varrho^{2}(U(S, \nu, 0)-U(S, \nu, \tau))\right)},
$$

where $\varrho$ is the non-negative penalty parameter.

In this case, the boundary conditions for the American call option are given by:

$$
\begin{aligned}
& U(S, \nu, \tau) \approx 0, \quad S \rightarrow 0, \\
& U(S, \nu, \tau) \approx \max \left\{S e^{-q \tau}-E e^{-r \tau}, S-E\right\}, \quad S \rightarrow+\infty, \\
& \frac{\partial U(S, \nu, \tau)}{\partial \nu} \approx 0, \quad \nu \rightarrow+\infty .
\end{aligned}
$$

\section{A new local RBF method}

A key feature of a meshless method is that it does not require a grid and only makes use of a scattered set of collocation points regardless of the connectivity information between the collocation points. The RBF approximation uses the pairwise distances between points alongside shape parameter. Distances are easily computed in any number of spatial dimensions, thus, working in higher dimensions does not increase the complexity of the method.

The meshfree RBF collocation technique for the solution of PDEs is similar to the spectral approach, i.e., we approximate the solution $P$ corresponding to $M$ spatial variables $\mathbf{y}=\left(y_{1}, \ldots, y_{M}\right)$ and time $\tau$ by using $N$ supporting nodes as follows:

$$
P(\mathbf{y}, \tau)=\sum_{j=1}^{N} \alpha_{j}(\tau) \phi\left(\left\|\mathbf{y}-\mathbf{y}^{j}\right\|\right),
$$

where time and space have been decoupled while we are using the Euclidean norm. Here centers $\mathbf{y}^{j}$ form a discretization of the computational domain.

The coefficients $\alpha_{j}(\tau)$ have to be determined by imposing that $P(\mathbf{y}, \tau)$ satisfies the equation at the centers $\mathbf{y}^{j}$. The radial function $\phi(\|\cdot\|)$ determines the approximation space as the span of the functions $\left\{\phi\left(\left\|\cdot-\mathbf{y}^{1}\right\|\right), \ldots\right.$, $\left.\phi\left(\left\|\cdot-\mathbf{y}^{N}\right\|\right)\right\}$. Here, we consider that $N=m \times n$, where $m$ and $n$ are the number nodes along the spatial variables $S$ and $\nu$, respectively. 
The technique applied in this section is a semi-discretization scheme at which all the spatial variables are discretized and the temporal variable stay intact in order to construct a set of ODEs. This system will then be solved using time-stepping methods.

In [38] Wendland constructed a popular family of compactly supported radial functions $\phi_{s, k}$ of the smoothness degree $2 k$ by starting with the truncated power function (which we know to be strictly positive definite) and radial on $\mathbb{R}^{M}$ for $M \geq 1$, and then walking through dimensions.

Here, we need the solution of the PIDEs to be two times differentiable along the spatial variables, hence one way is to apply the Wendland type $\phi_{3,2}$ function given by

$$
\phi_{3,2}(d)=\left(1-\frac{d}{c}\right)_{+}^{6}\left(3+18 \frac{d}{c}+35\left(\frac{d}{c}\right)^{2}\right),
$$

which its degree of smoothness is $C^{4}$ for any dimensions $M \leq 3$. Here the shape parameter $c>0$ controls the flatness and $d \geq 0$ is the radius (also known as the distance between two points in $\mathbb{R}^{2}$ ), i.e., $d=\|x-y\|_{2}=$ $\sqrt{\left(x_{1}-x_{2}\right)^{2}+\left(y_{1}-y_{2}\right)^{2}}$. In addition, $(\cdot)_{+}=\max \{\cdot, 0\}$. For the sake of simplicity, $\phi_{3,2}$ is denoted by $\phi(x, y)$ from now on. The choice and the motivation of using eqn (12) is that the function $\phi$ should be local in order to produce sparse interpolation matrices, can be applied for problems of dimensions $M \leq 3$ which is in our case a 2D PIDE problem, and also be smooth enough since we have second-order derivative of a function in the structure of (2). There are of course some other choices of $\phi$ from the Wendland family of function, but this one also has a low computational cost in terms of computing the function at each supporting node as well.

The numerical domain for (2) is basically $(S, \nu, \tau) \in \Omega \times(0, T]$, where

$$
\Omega=[0,+\infty) \times[0,+\infty) .
$$

The state space is unbounded in our problem. So, in order to be able to solve the model computationally we need to localize it to a bounded computational domain. However since the problem is well-posed, the computational domain in practice is considered to be bounded $\left(\Omega=\left[S_{\min }, S_{\max }\right] \times[0,1]\right)$, for example, $\Omega=[0,3 E] \times[0,1]$ or $\Omega=[0,4 E] \times[0,1]$. This justifies the use of essentially arbitrary artificial boundary conditions. Here for our local RBF scheme and unlike the FD method, in order to avoid constructing and handling ill-conditioned evaluation matrices, we apply the following transformation

$$
s=\frac{S-S_{\min }}{S_{\max }-S_{\min }}, \quad s \in[0,1]
$$

so as to turn the computational domain into the square $[0,1] \times[0,1]$. The original PIDE (2) does not change much and can be written as comes next:

$$
\begin{aligned}
\frac{\partial P}{\partial \tau}= & \frac{1}{2} \nu s^{2} \frac{\partial^{2} P}{\partial s^{2}}+\rho \sigma \nu s \frac{\partial^{2} P}{\partial s \partial \nu}+\frac{1}{2} \sigma^{2} \nu \frac{\partial^{2} P}{\partial \nu^{2}}+(r-q-\lambda \xi) s \frac{\partial P}{\partial s} \\
& +\kappa(\theta-\nu) \frac{\partial P}{\partial \nu}-(r+\lambda) P+\lambda \int_{0}^{\infty} P(s \eta, \nu, \tau) f(\eta) d \eta \\
= & L(P)+\lambda I(P),
\end{aligned}
$$

wherein $L(P)$, and $\lambda I(P)$ stand for the differential part (including the $(r+\lambda) P$ ) and the integral part of the model. This would change the initial condition, e.g., for the call option, we have

$$
P(s, \nu, 0)=\max \left\{\left(S_{\min }+\left(S_{\max }-S_{\min }\right) s\right)-E, 0\right\} .
$$

The PDE problem is well posed as an initial value (Cauchy) problem in at least one dimension. We assume that at time $\tau$, the exact solution of $(15)$, i.e., $P(s, \nu, \tau)$ is approximated via $p_{i, j}(\tau)$. To proceed, it is first observed that the solution (11) yields in the following functional approximations for the first temporal derivative, the first spatial derivative in the directions $s, \nu$, the second spatial derivative in the directions $s, \nu$, and the mixed derivative, respectively: $P^{(0,0,1)}(s, \nu, \tau)=\sum_{j=1}^{N} \alpha_{j}^{\prime}(\tau) \phi\left(\left\|\mathbf{y}-\mathbf{y}^{j}\right\|\right), P^{(1,0,0)}(s, \nu, \tau)=\sum_{j=1}^{N} \alpha_{j}(\tau) \phi^{(1,0,0)}\left(\left\|\mathbf{y}-\mathbf{y}^{j}\right\|\right)$, 
$P^{(0,1,0)}(s, \nu, \tau)=\sum_{j=1}^{N} \alpha_{j}(\tau) \phi^{(0,1,0)}\left(\left\|\mathbf{y}-\mathbf{y}^{j}\right\|\right), P^{(2,0,0)}(s, \nu, \tau)=\sum_{j=1}^{N} \alpha_{j}(\tau) \phi^{(2,0,0)}\left(\left\|\mathbf{y}-\mathbf{y}^{j}\right\|\right), P^{(0,2,0)}(s, \nu, \tau)$ $=\sum_{j=1}^{N} \alpha_{j}(\tau) \phi^{(0,2,0)}\left(\left\|\mathbf{y}-\mathbf{y}^{j}\right\|\right), P^{(1,1,0)}(s, \nu, \tau)=\sum_{j=1}^{N} \alpha_{j}(\tau) \phi^{(1,1,0)}\left(\left\|\mathbf{y}-\mathbf{y}^{j}\right\|\right)$.

To approximate the differential part $L(P)$ of the Bates model using the local Wendland RBF (12), we need the partial derivatives including the shape parameter $c$ to be determined. After several algebraic calculation, these partial derivatives are given as follows:

$$
\begin{aligned}
\phi_{s}(x, y) & =-\frac{56(c-d)_{+}^{5}(c+5 d)\left(x_{1}-x_{2}\right)}{c^{8}}, \\
\phi_{s, s}(x, y) & =-\frac{56(c-d)_{+}^{4}\left(c^{2}+4 c d-35 d^{2}+30\left(y_{1}-y_{2}\right)^{2}\right)}{c^{8}}, \\
\phi_{\nu}(x, y) & =-\frac{56(c-d)_{+}^{5}(c+5 d)\left(y_{1}-y_{2}\right)}{c^{8}}, \\
\phi_{\nu, \nu}(x, y) & =-\frac{56(c-d)_{+}^{4}\left(c^{2}+4 c d-35 d^{2}+30\left(x_{1}-x_{2}\right)^{2}\right)}{c^{8}}, \\
\phi_{s, \nu}(x, y) & =\frac{1680(c-d)_{+}^{4}\left(x_{1}-x_{2}\right)\left(y_{1}-y_{2}\right)}{c^{8}} .
\end{aligned}
$$

Now, we are able to define the following elements of the $N \times N$ matrices $(1 \leq i \leq m, 1 \leq j \leq n)$ :

$$
\begin{array}{lr}
(\boldsymbol{\Phi})_{i, j}=\phi\left(x_{i}, y_{j}\right), & \left(\mathbf{\Phi}_{s}\right)_{i, j}=\phi_{s}\left(x_{i}, y_{j}\right), \\
\left(\boldsymbol{\Phi}_{s, s}\right)_{i, j}=\phi_{s, s}\left(x_{i}, y_{j}\right), & \left(\mathbf{\Phi}_{\nu}\right)_{i, j}=\phi_{\nu}\left(x_{i}, y_{j}\right), \\
\left(\boldsymbol{\Phi}_{\nu, \nu}\right)_{i, j}=\phi_{\nu, \nu}\left(x_{i}, y_{j}\right), & \left(\boldsymbol{\Phi}_{s, \nu}\right)_{i, j}=\phi_{s, \nu}\left(x_{i}, y_{j}\right),
\end{array}
$$

Note that the matrix $\mathbf{\Phi}$ is known as the evaluation or the interpolation matrix in the meshfree RBF methodology. Substituting equations (22)-(24) based on (17)-(21) into (15), results in the following matrix equation:

$$
\frac{\partial p(\tau)}{\partial \tau}=\mathbf{B} p(\tau)
$$

wherein

$$
p(\tau)=\left(p_{1,1}(\tau), p_{1,2}(\tau), \ldots, p_{1, n}(\tau), p_{2,1}(\tau), \ldots, p_{m, n}(\tau)\right)^{*}
$$

and

$$
\mathbf{B}=\left[\frac{1}{2} \boldsymbol{\nu} \mathbf{s}^{2} \boldsymbol{\Phi}_{s, s}+\rho \sigma \boldsymbol{\nu} \mathbf{s} \boldsymbol{\Phi}_{s, \nu}+\frac{1}{2} \sigma^{2} \boldsymbol{\nu} \boldsymbol{\Phi}_{\nu, \nu}+(r-q-\lambda \xi) \mathbf{s} \boldsymbol{\Phi}_{s}+\kappa(\theta I-\boldsymbol{\nu}) \mathbf{\Phi}_{\nu}-(r+\lambda) I\right] \boldsymbol{\Phi}^{-1},
$$

and $I=I_{N \times N}$ is the identity matrix while s and $\boldsymbol{\nu}$ (shown in bold) are diagonal matrices of the same size in the $s$ and $\nu$ directions containing the grids in these directions. Note that the influence of the jump in the Bates model has not been incorporated into the set of ODEs (25) yet. This would be pursued in the next section.

To be more precise regarding (25), the collocation is made locally over a set of overlapping domains of influence and the time-stepping is performed in an explicit way. Only systems of equations of low dimensions must be solved which is a strong point of the local meshfree RBF method in contrast to the standard FD scheme using uniform grids which mainly leads to ODEs system of high sizes.

As pointed out in [27], if $\boldsymbol{\Phi}$ is invertible, then the equation (25) has a solution. In this work and as already did in [19], we use the Moore-Penrose inverse of the evaluation matrices so as to avoid any ill-conditioning in the computation of $\boldsymbol{\Phi}^{-1}$. Therefore, as long as the Moore-Penrose inverse exists, our local RBF approach for the Bates model exists too.

It is remarked that an important attention should be focused on the choice of the shape parameter in our new local RBF method (25). To be more precise, unlike other conventional methods such as FD and FE, stability of meshless methods also depend on the value of shape parameter $c$ as well as the total numbers of supporting nodes $N$ in the working domain. Figures 1-2 show the plot of the local Wendland RBF (12) using different parameters in the domain of validity $r=\|x-y\|_{2} \leq c \times L$, where basically we choose $L=\tau$. 

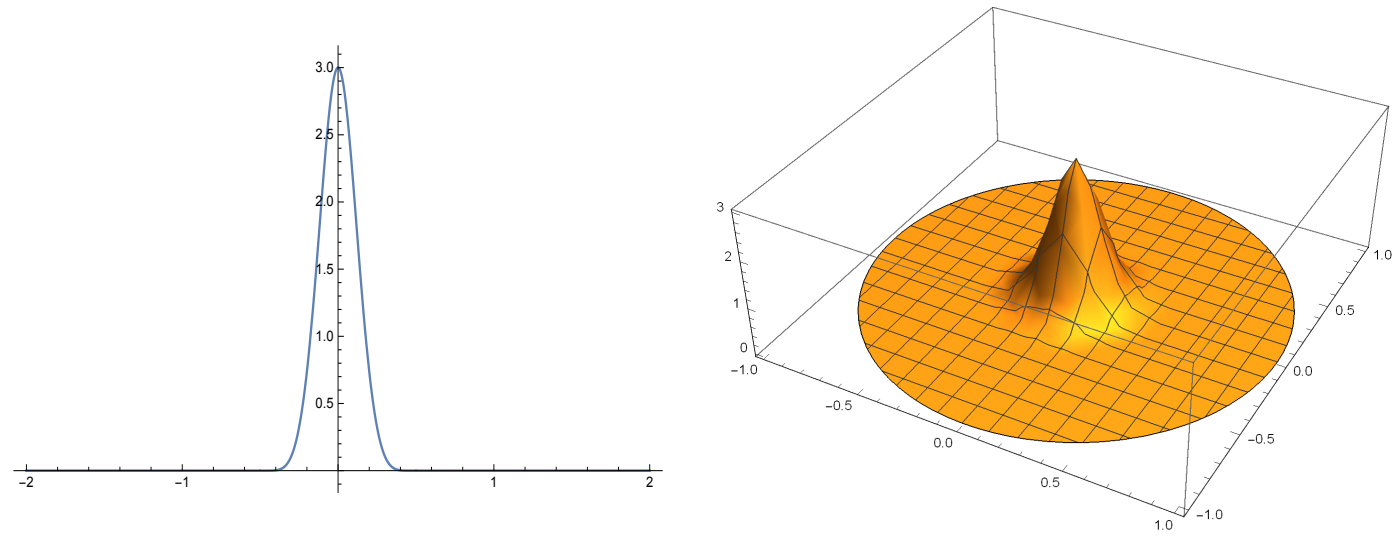

Figure 1: One- and two-dimensional local Wendland RBF (12) for $c=0.5$ and $L=2$.
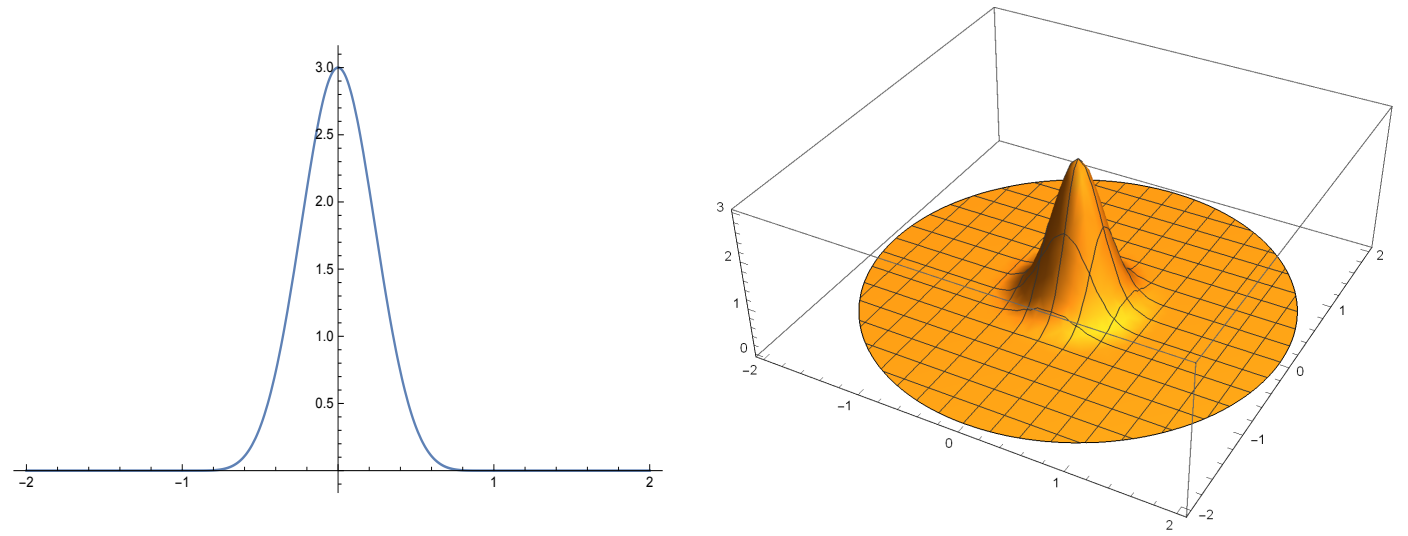

Figure 2: One- and two-dimensional local Wendland RBF (12) for $c=1$ and $L=2$.

\section{Discretization of the integral operator}

The jump-integral term has the form of a convolution product, and hence could be efficiently evaluated using a numerical rule. The fast Fourier transform applied by Andersen and Andreasen [3] could allow the user to compute the jump-integral term but the use of the fast Fourier transform introduces additional localization and interpolation error, specially if it is employed in conjunction with a non-uniform spatial mesh, so the discretization of the integral operator must be performed on a mesh which is finer than the mesh used for the discretization of the differential operators. Hence, it would be requisite to approximate the integral term using the computational nodal points which have already been used and applied in the process of discretization of the differential part $L(P)$.

Herein, the nonlocal integral operator in (15), i.e.,

$$
I(P)=\int_{0}^{\infty} P(s \eta, \nu, \tau) f(\eta) d \eta
$$

is discretized using the linear interpolation for $P(s \eta, \nu, \tau)$ among the grid points, which is an extension over the discussion in [32]. A change of variable

$$
z=s \eta
$$


for the integral part only is taken into account to transform it into

$$
I(P)=\int_{0}^{\infty} P(z, \nu, \tau) f\left(\frac{z}{s}\right)\left(\frac{1}{s}\right) d z .
$$

Now, by using linear interpolation we get an approximation

$$
I_{i}(P) \approx \sum_{l=1}^{m-1} M_{i, l},
$$

of $I$ at each grid point $s_{i}, i=2, \ldots, m-1$, where

$$
M_{i, l}=\int_{s_{l}}^{s_{l+1}}\left(\frac{s_{l+1}-z}{\Delta_{l}} P\left(s_{l}, \nu, \tau\right)+\frac{z-s_{l}}{\Delta_{l}} P\left(s_{l+1}, \nu, \tau\right)\right) f\left(\frac{z}{s_{i}}\right)\left(\frac{1}{s_{i}}\right) d z,
$$

wherein $\Delta_{l}=s_{l+1}-s_{l}$ is the step size. Taking into consideration the log-normal distribution $f(\eta)$ based on (3), one may obtain

$$
\begin{aligned}
M_{i, l}= & \int_{s_{l}}^{s_{l+1}}\left(\frac{s_{l+1}-z}{\Delta_{l}} P\left(s_{l}, \nu, \tau\right)+\frac{z-s_{l}}{\Delta_{l}} P\left(s_{l+1}, \nu, \tau\right)\right) \\
& \times \exp \left[-\frac{\left(\ln \left(\frac{z}{s_{i}}\right)-\left(\gamma-\frac{1}{2} \hat{\sigma}^{2}\right)\right)^{2}}{2 \hat{\sigma}^{2}}\right]\left(\frac{1}{s_{i}}\right) d z \\
= & \frac{1}{\sqrt{2 \pi} \hat{\sigma}} \int_{s_{l}}^{s_{l+1}}\left(\frac{s_{l+1}-z}{\Delta_{l}} P\left(s_{l}, \nu, \tau\right)+\frac{z-s_{l}}{\Delta_{l}} P\left(s_{l+1}, \nu, \tau\right)\right) \\
& \times \exp \left[-\frac{\left(\ln \left(\frac{z}{s_{i}}\right)-\left(\gamma-\frac{1}{2} \hat{\sigma}^{2}\right)\right)^{2}}{2 \hat{\sigma}^{2}}\right] / z d z .
\end{aligned}
$$

The proper integral (33) can be computed explicitly. It yields in

$$
\begin{aligned}
M_{i, l}= & -\frac{1}{2 \Delta_{l}}\left(\operatorname { e x p } ( \gamma ) \left(\operatorname{erf}\left(\frac{-2 \ln \left(\frac{1}{s_{i}}\right)-2 \ln \left(s_{l}\right)+2 \gamma+\hat{\sigma}^{2}}{2 \sqrt{2} \hat{\sigma}}\right)\right.\right. \\
& \left.-\operatorname{erf}\left(\frac{-2 \ln \left(\frac{s_{l+1}}{s_{i}}\right)+2 \gamma+\hat{\sigma}^{2}}{2 \sqrt{2} \hat{\sigma}}\right)\right) s_{i}\left(P\left(s_{l}, v, \tau\right)-P\left(s_{l+1}, v, \tau\right)\right) \\
& +\left(\operatorname{erf}\left(\frac{2 \ln \left(\frac{1}{s_{i}}\right)+2 \ln \left(s_{l}\right)-2 \gamma+\hat{\sigma}^{2}}{2 \sqrt{2} \hat{\sigma}}\right)\right. \\
& \left.\left.-\operatorname{erf}\left(\frac{2 \ln \left(\frac{s_{l+1}}{s_{i}}\right)-2 \gamma+\hat{\sigma}^{2}}{2 \sqrt{2} \hat{\sigma}}\right)\right)\left(s_{l+1} P\left(s_{l}, v, \tau\right)-s_{l} P\left(s_{l+1}, v, \tau\right)\right)\right),
\end{aligned}
$$

wherein the error function $\operatorname{erf}(\cdot)$ is the integral of the Gaussian distribution and is defined by

$$
\operatorname{erf}(z)=\frac{2}{\sqrt{\pi}} \int_{0}^{z} \exp \left(-t^{2}\right) d t
$$


The expression given in (34) can further be simplified as follows:

$$
\begin{gathered}
M_{i, l}=\frac{1}{2 \Delta_{l}}\left(s_{i} \alpha_{l}\left(\operatorname{erfc}\left(\frac{-2 \ln \left(\frac{s_{l}}{s_{i}}\right)+2 \gamma+\hat{\sigma}^{2}}{2 \sqrt{2} \hat{\sigma}}\right)-\operatorname{erfc}\left(\frac{-2 \ln \left(\frac{s_{l+1}}{s_{i}}\right)+2 \gamma+\hat{\sigma}^{2}}{2 \sqrt{2} \hat{\sigma}}\right)\right)\right. \\
\left.+\beta_{l}\left(\operatorname{erfc}\left(\frac{2 \ln \left(\frac{s_{l}}{s_{i}}\right)-2 \gamma+\hat{\sigma}^{2}}{2 \sqrt{2} \hat{\sigma}}\right)-\operatorname{erfc}\left(\frac{2 \ln \left(\frac{s_{l+1}}{s_{i}}\right)-2 \gamma+\hat{\sigma}^{2}}{2 \sqrt{2} \hat{\sigma}}\right)\right)\right),
\end{gathered}
$$

wherein $\alpha_{l}=\exp (\gamma)\left(P\left(s_{l}, v, \tau\right)-P\left(s_{l+1}, v, \tau\right)\right), \beta_{l}=s_{l+1} P\left(s_{l}, v, \tau\right)-s_{l} P\left(s_{l+1}, v, \tau\right)$ and the complementary error function $\operatorname{erfc}(\cdot)$ is expressed as comes next:

$$
\operatorname{erfc}(z)=1-\operatorname{erf}(z)
$$

Due to the integral term, the final semi-discretization leads to a full matrix. We remark that direct solution methods are usually expensive with a full matrix, and therefore other numerical methods should be considered. Furthermore, we should avoid constructing systems of higher sizes which is a derivation of applying FD and FE methods. But, using our new local RBF method, we are able to obtain a set of ODEs of the following form with a reasonable size:

$$
\frac{\partial p(\tau)}{\partial \tau}=\mathbf{B} p(\tau)+\lambda \mathbf{I},
$$

where $\mathbf{I}$ is a vector of dimension $N \times 1$ containing the integral approximations at each nodes. Each component of I could consist of at least several unknowns in terms of $p_{i, j}(\tau)$.

Simplifying (38) would result in the following set of ODEs:

$$
\frac{\partial p(\tau)}{\partial \tau}=\overline{\mathbf{B}} p(\tau)
$$

Finally, by imposing the boundaries (and by considering the penalty approach (9) for the American-style option) we obtain the following system of semi-discretized ODEs subject to a non-smooth initial condition:

$$
\left\{\begin{array}{l}
\frac{\partial p(\tau)}{\partial \tau}=\tilde{\mathbf{B}} p(\tau)+b(\tau)+F(p(\tau)), \\
p(s, \nu, 0)=p_{0} \text { is given, }
\end{array}\right.
$$

where $b(\tau)$ is a vector corresponds to the incorporation of the boundary at $s \rightarrow+\infty$. Furthermore, it is stressed that the first $n$ rows and the last $n$ rows of $\tilde{\mathbf{B}}$ would always be zero due to incorporation of the boundaries for the spatial variable $s$.

\section{Time discretization}

Different time integration techniques can now be applied. In the accompanying discourse, we provide different time stepping methods that could be applied for solving the locally well-posed semi-discretized system of ODEs (40) after discretization of the PIDEs. Well-posedness means that there exists a unique solution (depending on the initial condition) which satisfy (40). As a matter of fact, we assume that $G(\tau, p(\tau))=\tilde{\mathbf{B}} p(\tau)+b(\tau)+F(p(\tau))$ is continuous in its first argument, $\tau$, and locally uniformly Lipschitz continuous in its second argument.

The exact solution of the set of ODEs (40) is given by [16, Section 2.1]:

$$
P(\tau)=\exp (\tilde{\mathbf{B}} \tau) p_{0}+\int_{0}^{\tau} \exp (\tilde{\mathbf{B}}(\tau-\varrho))[b(\varrho)+F(p(\varrho))] d \varrho .
$$

The restrictions occurred in the process of computing matrix exponential and particularly the Volterra integral in (41) makes the users to apply several different time-stepping strategies to solve 40. 
Let us introduce the temporal discretization with fixed time step $\Delta_{\tau}=\frac{\tau}{k-1}$, so $\tau_{\iota}=(\iota-1) \Delta_{\tau}, \iota=1, \ldots, k$. The explicit Euler method which is a first-order numerical procedure for solving system of ODEs with a given initial value is given by

$$
p\left(\tau_{\iota+1}\right)=p\left(\tau_{\iota}\right)+\Delta_{\tau} G\left(\tau_{\iota}, p\left(\tau_{\iota}\right)\right)
$$

where

$$
G\left(\tau_{\iota}, p\left(\tau_{\iota}\right)\right)=\tilde{\mathbf{B}} p\left(\tau_{\iota}\right)+b\left(\tau_{\iota}\right)+F\left(p\left(\tau_{\iota}\right)\right) .
$$

We are often interested in the behavior of a time discretization method as the discretization parameter $\Delta_{\tau}$ decreases to zero. When it is required for clarity in such situations, we will write $p_{k}$ to indicate an approximate solution obtained via a time-stepping method using $\Delta_{\tau}$ for the exact value $P_{\text {exact }}(\tau)$. The scheme (42) converges to the solution since it satisfies

$$
\left\|p_{k}-P_{\text {exact }}(\tau)\right\| \rightarrow 0, \quad k \rightarrow+\infty .
$$

The method (42) is quite straight-forward for implementations but it requires a large number of steps to converge. This entails a high computational cost. Hence, an alternative known as the explicit midpoint method, that is, a one-step method can be used for solving our large system of ODEs (particularly in the nonlinear cases due to applying the penalty approach (9) for tackling American options) as follows [34, p. 328]:

$$
p\left(\tau_{\iota+1}\right)=p\left(\tau_{\iota}\right)+\Delta_{\tau} G\left(\tau_{\iota}+\frac{\Delta_{\tau}}{2}, \varphi_{\iota}\right),
$$

where

$$
\varphi_{\iota}=p\left(\tau_{\iota}\right)+\frac{\Delta_{\tau}}{2} G\left(\tau_{\iota}, p\left(\tau_{\iota}\right)\right) .
$$

The explicit midpoint method is also known as the modified Euler method. The name of the method comes from the fact that in (45) the function $G$ (giving the slope of the solution) is evaluated at

$$
\tau=\tau_{\iota}+\frac{\Delta_{\tau}}{2},
$$

which is the midpoint between $\tau_{\iota}$ at which the value of $p(\tau)$ is known and $\tau_{\iota+1}$ at which the value of $p(\tau)$ needs to be found.

The local error at each step of the midpoint method is of order $\mathcal{O}\left(\Delta_{\tau}^{3}\right)$, giving a global error of order $\mathcal{O}\left(\Delta_{\tau}^{2}\right)$. Thus, while more computationally intensive than Euler's method, the midpoint method's error generally decreases faster as $\Delta_{\tau} \rightarrow 0$.

Theorem 5.1. Let the function $G\left(\tau_{\iota}, p\left(\tau_{\iota}\right)\right)=\tilde{\mathbf{B}} p\left(\tau_{\iota}\right)+b\left(\tau_{\iota}\right)+F\left(p\left(\tau_{\iota}\right)\right)=\tilde{\mathbf{B}} p\left(\tau_{\iota}\right)+H\left(\tau_{\iota}, p\left(\tau_{\iota}\right)\right)$ satisfies the Lipschitz condition in its second argument, while all the eigenvalues of $\tilde{\mathbf{B}}$ lie in the left half plane and there exists a basis of $R^{N}$ consisting of eigenvectors of $\tilde{\mathbf{B}}$. Then, the constructed proposed scheme for pricing (2), via the semi-discretized system of ODEs (40)E( $(45)$ is conditionally time-stable.

Proof. Satisfying the Lipschitz condition provides that (40) has a unique solution. Now, the time-stepping solver (45) for tackling (40) (after imposing the boundaries) can be written as:

$$
p_{\iota+1}=\left(I+\Delta_{\tau} \tilde{\mathbf{B}}+\frac{\left(\Delta_{\tau} \tilde{\mathbf{B}}\right)^{2}}{2}\right) p_{\iota}+\Delta_{\tau}\left(b\left(\tau_{\iota}+\frac{\Delta_{\tau}}{2}\right)+F\left(\varphi_{\iota}\right)\right) .
$$

Using the Linearization theorem on the function $H$, the stability of the difference equation (48) is reduced to study the eigenvalues of the coefficient matrix of $p_{\iota}$. Now, if we let $p_{\iota}$ be expanded in the eigen-basis (say, $u_{1}, u_{2}$, $\left.\cdots, u_{N}\right)$ of the real matrix $\tilde{\mathbf{B}}$, we may write:

$$
p_{\iota}=\alpha_{1}^{(\iota)} u_{1}+\alpha_{2}^{(\iota)} u_{2}+\cdots+\alpha_{N}^{(\iota)} u_{N}
$$


If we now apply the modified Euler's method, we find

$$
\begin{aligned}
p_{\iota+1}= & \left(I+\Delta_{\tau} \tilde{\mathbf{B}}+\frac{\left(\Delta_{\tau} \tilde{\mathbf{B}}\right)^{2}}{2}\right)\left(\alpha_{1}^{(\iota)} u_{1}+\alpha_{2}^{(\iota)} u_{2}+\cdots+\alpha_{N}^{(\iota)} u_{N}\right) \\
= & \alpha_{1}^{(\iota)}\left(I+\Delta_{\tau} \tilde{\mathbf{B}}+\frac{\left(\Delta_{\tau} \tilde{\mathbf{B}}\right)^{2}}{2}\right) u_{1}+\alpha_{2}^{(\iota)}\left(I+\Delta_{\tau} \tilde{\mathbf{B}}+\frac{\left(\Delta_{\tau} \tilde{\mathbf{B}}\right)^{2}}{2}\right) u_{2} \\
& +\cdots+\alpha_{N}^{(\iota)}\left(I+\Delta_{\tau} \tilde{\mathbf{B}}+\frac{\left(\Delta_{\tau} \tilde{\mathbf{B}}\right)^{2}}{2}\right) u_{N} .
\end{aligned}
$$

Now, as $u_{i}$ is an eigenvector of $\tilde{\mathbf{B}}$, we have

$$
\begin{aligned}
\left(I+\Delta_{\tau} \tilde{\mathbf{B}}+\frac{\left(\Delta_{\tau} \tilde{\mathbf{B}}\right)^{2}}{2}\right) u_{i} & =u_{i}+\Delta_{\tau} \tilde{\mathbf{B}} u_{i}+\frac{\left(\Delta_{\tau} \tilde{\mathbf{B}}\right)^{2}}{2} u_{i} \\
& =u_{i}+\Delta_{\tau} \omega_{i} u_{i}+\frac{\left(\Delta_{\tau} \omega_{i}\right)^{2}}{2} u_{i} \\
& =\left(1+\Delta_{\tau} \omega_{i}+\frac{\left(\Delta_{\tau} \omega_{i}\right)^{2}}{2}\right) u_{i}
\end{aligned}
$$

where $\omega_{i}$ for any $1 \leq i \leq N$ is the eigenvalue of the matrix $\tilde{\mathbf{B}}$ associated with the eigenvector $u_{i}$. The relation (51) implies that

$$
p_{\iota+1}=\sum_{i=1}^{N} \alpha_{i}^{(\iota)}\left(1+\Delta_{\tau} \omega_{i}+\frac{\left(\Delta_{\tau} \omega_{i}\right)^{2}}{2}\right) u_{i} .
$$

Using the uniqueness of the basis representation, it is straightforward to write

$$
\alpha_{i}^{(\iota+1)}=\left(1+\Delta_{\tau} \omega_{i}+\frac{\left(\Delta_{\tau} \omega_{i}\right)^{2}}{2}\right) \alpha_{i}^{(\iota)} .
$$

Accordingly, the time-stability is reduced to

$$
\left|1+\Delta_{\tau} \omega_{i}+\frac{\left(\Delta_{\tau} \omega_{i}\right)^{2}}{2}\right| \leq 1, \quad i=1,2, \ldots, N,
$$

which is equal to the scalar relationship based on the eigenvalues of the matrix $\tilde{\mathbf{B}}$. Solving (54) based on the fact that $\Delta_{\tau}>0$ yields the following bounds for the real and imaginary parts of the eigenvalues:

$$
-\frac{2}{\Delta_{\tau}} \leq \operatorname{Re}\left(\omega_{i}\right) \leq 0
$$

and

$$
-\Xi_{i} \leq \operatorname{Im}\left(\omega_{i}\right) \leq \Xi_{i},
$$

wherein $\operatorname{Re}(\cdot)$ and $\operatorname{Im}(\cdot)$ are the real and imaginary parts, while

$$
\Xi_{i}=\sqrt{2 \sqrt{-\frac{\operatorname{Re}\left(\omega_{i}\right)\left(\Delta_{\tau} \operatorname{Re}\left(\omega_{i}\right)+2\right)}{\Delta_{\tau}^{3}}}-\frac{\operatorname{Re}\left(\omega_{i}\right)\left(\Delta_{\tau} \operatorname{Re}\left(\omega_{i}\right)+2\right)}{\Delta_{\tau}}}
$$

Thus, the constructed scheme is stable if the temporal step size $\Delta_{\tau}$ satisfy (55) and (56) for any eigenvalues of $\tilde{\mathbf{B}}$. Using the polar representation $\omega_{\varsigma}=r_{\varsigma} \exp \left(i \theta_{\varsigma}\right)$, then (54) can be written as

$$
\Lambda\left(r_{\varsigma}, \theta_{\varsigma}, \Delta_{\tau}\right) \leq 0, \quad 1 \leq \varsigma \leq N,
$$


wherein $\Lambda(r, \theta, \xi)=\frac{r^{4}}{4} \xi^{3}+r^{3} \cos (\theta) \xi^{2}+2 r^{2} \cos ^{2}(\theta) \xi+2 r \cos (\theta)$. Subsequently, the condition (54) is satisfied if the real part of all the eigenvalues of $\tilde{\mathbf{B}}$ is non-positive and $\Delta_{\tau} \leq \xi_{0}$, where $\xi_{0}=\min _{1 \leq \varsigma \leq N}\left\{\xi_{\varsigma}\right\}$ and $\xi_{\varsigma}$ is the unique positive solution of $\Lambda\left(r_{\varsigma}, \theta_{\varsigma}, \xi\right)=0$. For numerical purposes, by taking into account the nature of the matrix $\tilde{\mathbf{B}}$, cf. Figure 5, wherein the dominance of the real part for large eigenvalues is prevalent, a fair time-stability bound can be derived by

$$
\Delta_{\tau}<\frac{-2}{\operatorname{Re}\left(\omega_{\max }^{\tilde{\mathrm{B}}}\right)},
$$

where $\omega_{\max }^{\tilde{\mathrm{B}}}$ stands for the largest eigenvalue of $\tilde{\mathbf{B}}$. An important remark here is that this conditional time stability is clearly dependent on the choice of the shape parameter, since its choice has clear impact on the largest eigenvalues of $\tilde{\mathbf{B}}$. Therefore, the scheme is stable (in the Lyapunov sense) under (59). The proof is now complete.

The convergence of the whole numerical procedure, i.e.,

$$
\left\|p_{m, n, k}-P_{\text {exact }}(s, \nu, \tau)\right\| \rightarrow 0,
$$

can now be hold under the conditional stability condition (59) when the all step sizes end to zero which imply the consistency as well.

\section{Numerical experiments}

In this section, we consider numerical experiments on three benchmark problems and assess the actual convergence behavior of the FD and our new RBF collocation methods for different cases of the Bates PIDE. A comparison with the Monte-Carlo method and Fourier Transform (FT) approach is also given (in different cases). For any given numbers of mesh points $m, n$ in the $s$-and $\nu$-directions, we compute the value of European and American options for different types of methods and time-stepping schemes.

The discretization along the price variable, also known as spatial discretization, is carried out by the standard (centered) three-point FD scheme, then it means that when the spatial discretization interval is halved, the error is reduced by a factor approximately equal to four [15].

The simulations are done in Mathematica 11.0 with built-in precision [5] while we have set AccuracyGoal $\rightarrow 5$, PrecisionGoal $\rightarrow 5$ in the codes to run the programs (for the part of solving set of ODEs as quickly as possible). To emphasize the performance of the proposed computational procedure, all the simulations are run on an office laptop with Windows 7 Ultimate equipped Intel(R) Core(TM) i5-2430M CPU 2.40GHz processor and $16.00 \mathrm{~GB}$ of RAM on a 64-bit operating system.

The computer time needed to obtain the option prices will be denoted as CPUTime in seconds. We apply different strategies for choosing the free positive shape parameter in order to put on show the reliability of the

proposed local WRBF method. Moreover, let WRBF denote the approximation method based on the proposed local RBF approach.

An equidistant computational grid in each direction is considered, though any types of mesh could be used for our local WRBF method. Furthermore, to have a fair comparisons, we applied (42) for time stepping of the European options and (45) for time stepping of the American options. Furthermore, for the nodes locating at the boundary $\nu=0$, we impose no boundary and we take such nodes account as interior node at which the PIDE without the integral term satisfy. That is, the semi-discretized equations of the PIDE (without the integral term) are considered to be valid at this boundary.

The following error measure

$$
\left\|p_{m, n, k}\left(s_{i}, \nu_{j}, \tau_{\iota}\right)-P_{\text {ref }}(s, \nu, \tau)\right\|,
$$

is used for option comparisons. Since the exact solutions are mostly not available, then a very accurate estimation with a large number of nodes in both the time and space directions is taken into consideration from the literature.

Test Problem 6.1. [25] In this test, we compare the results of different methods in the European call case applying the following parameters

$$
\tau=1, E=100, r=0.025, q=0, \theta=0.04, \kappa=1.5, \sigma=0.3, \lambda=0, \rho=-0.9 .
$$




\begin{tabular}{|c|c|c|c|c|c|c|c|}
\hline Method & $m$ & $n$ & Size & $k$ & Price $U$ & Error & CPUTime \\
\hline FD & & & & & & & \\
& 20 & 20 & 400 & 401 & 8.70011 & $1.94763 \times 10^{-1}$ & 0.91 \\
& 40 & 25 & 1000 & 2001 & 8.59748 & $2.97389 \times 10^{-1}$ & 2.67 \\
& 40 & 40 & 1600 & 2001 & 8.67396 & $2.20911 \times 10^{-1}$ & 5.39 \\
& 65 & 45 & 2925 & 4001 & 8.86090 & $3.39711 \times 10^{-2}$ & 15.77 \\
\hline WRBF & & & & & & & \\
& 14 & 14 & 196 & 401 & 9.55241 & $6.57545 \times 10^{-1}$ & 1.09 \\
& 17 & 15 & 255 & 501 & 9.11440 & $2.19528 \times 10^{-1}$ & 2.26 \\
& 18 & 18 & 324 & 801 & 8.98740 & $9.25280 \times 10^{-2}$ & 4.57 \\
& 20 & 20 & 400 & 801 & 8.88861 & $6.26023 \times 10^{-3}$ & 7.80 \\
\hline
\end{tabular}

Table 1: Results of comparisons for Experiment 6.1.

For this scenario, we compare the price obtained through each scheme with the price obtained through the very refined implementation of Fourier Transform (FT) approach, i.e., 8.894869, which we took as the reference in the hot zone $(S, v)=(100,0.04)$, [25]. The computational domain for the FD is $\Omega=[0,3 E] \times[0,1]$ and its transformation to $\bar{\Omega}=[0,1] \times[0,1]$ for the WRBF.

One of the earliest techniques in order to suggest a shape parameter was proposed by Hardy in [23]. This technique is given by

$$
c=0.815 \theta, \quad \theta=\frac{1}{N} \sum_{i=1}^{N} \theta_{i},
$$

where $\theta_{i}$ is the distance of the $i$-th data point to its nearest neighbor. In this experiment we have applied $c=$ $(1 / 2) 0.815 \theta$ to include less number of supporting nodes in the process of building our matrices (22)-(27).

The results for this test are put together in Table 1. As we may see, the option price can be computed with a good level of accuracy in a short piece of time using WRBF while FD requires more and more spatial nodes in order to reach higher accuracies. Figure 3 displays the option price functions $P$ on the transformed domain $(s, v) \in[0,1] \times[0,1]$ using the WRBF. It shows that the WRBF solution is reliable and it does not contain any spurious of oscillation specially near the far field boundaries.

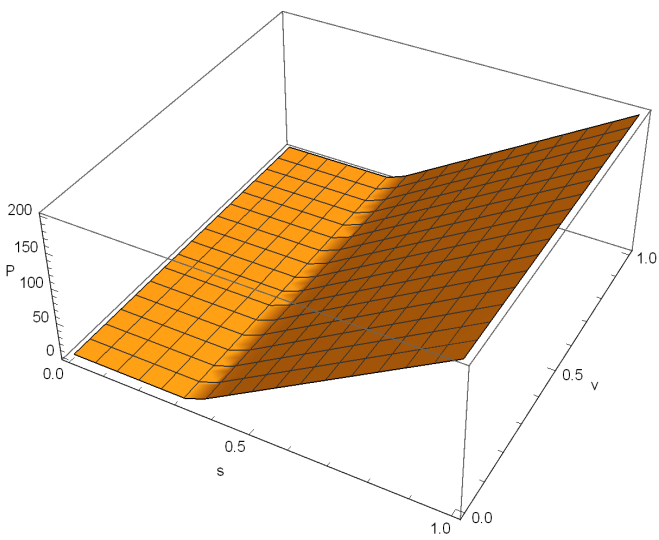

(a) $\tau=0$

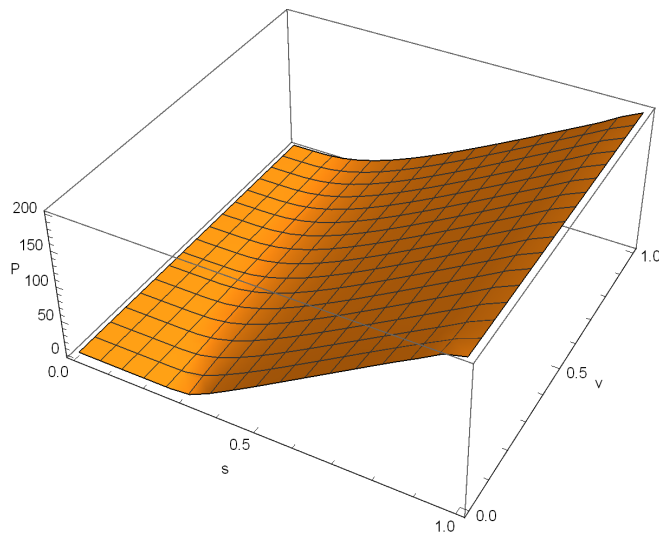

(b) $\tau=1$

Figure 3: Option price for Test Problem 6.1 with $m=20, n=20$ and $k=1001$, at (a) initial moment and (b) final moment.

The results are mostly reported at some certain points of the domain which are, also known as hot zones, (i.e., $(100,0.04))$ throughout this section. Actually, these values are deemed significant from the financial standpoint [6]. Furthermore, if the point that we are computing for, is not a node of the mesh, the approximate solution at such a point is obtained by a built-in interpolation command in the applied programming package Mathematica. 
To further validate the proposed approach, in the following, the approximated prices are compared with reference benchmarks computed using adaptive finite difference (AFD) and Monte Carlo methods (MC) [37]. The reference prices are brought forward in Table 2 based a giant computational grid $8193 \times 4097$ refined grid in space and 2048 time steps. The Monte Carlo prices are computed based on 268 million paths with 1000 time steps.

From Table 1 we can state that for a fixed absolute error, e.g., $10^{-3}$, FD requires more than $m=65, n=45$ points for spatial and $k=4001$ for temporal discretization, while for this accuracy we only need (around) $m=20, n=20$ and $k=801$, number points for discretizations, and thus $(65 \times 45) /(20 \times 20) \simeq 7$ times more spatial points and subsequently $15.77 / 7.80 \simeq 2$ more CPU times are required for FD in contrast to the WRBF.

\begin{tabular}{|c|c|c|c|}
\hline Option type & European Call, MC & European Call, AFD & American Call, AFD \\
\hline Price $U$ & 6.156860 & 6.157288 & 6.161108 \\
\hline
\end{tabular}

Table 2: Reference prices.

Test Problem 6.2. [37] The aim of this test is to study the variation of the resultant error for a European option under the Bates model. The parameters are selected as follows:

$$
\begin{aligned}
& \tau=0.5, E=100, r=0.02, q=0.06, \theta=0.04, \kappa=2.0, \\
& \sigma=0.25, \hat{\sigma}=0.4, \mu=-0.5, \lambda=0.2, \rho=-0.5 .
\end{aligned}
$$

Table 3 provides the numerical comparison and accuracies of different methods while the number of points along the spatial directions are increasing. The results show several choices for the number of time steps. The computational domain for the FD is $\Omega=[0,4 E] \times[0,1]$ (exactly based on [37]) and the transformation of $\Omega=$ $[0,3 E] \times[0,1]$ to $\Omega=[0,1] \times[0,1]$ for the WRBF.

Here and as we pointed out at the beginning of the section, we apply a different strategy known as "guessing" and fixing it for the shape parameter in all cases. Here, we have chosen $c=2$ and all the results are driven based on this for this experiment. The results declares that how our proposed WRBF scheme converge to the true benchmark price as quickly as possible and it reaches good accuracies in contrast to its correspondent FD scheme which require a higher number of computational supporting nodes to reach the same accuracies. To illustrate further, in Figure 4 (left), we have provided the computational grid which is equidistant in each direction for the case $m=n=26$.

Noting that the empty circles are the nodes at which PIDE (15) must be imposed so as to obtain the approximate solutions while the three known boundaries are given by filled circles. Although here we have applied an equidistant grid, the computational grid for our local method could of any type and structure due to applying the meshfree technology (this is unlike the FD scheme which is limited to the standard grids unless the finite difference approximations get applied for non-standard grid by accepting higher computational burden). Furthermore, the structure of the coefficient matrix $\tilde{\mathbf{B}}$ in (40) is illustrated in Figure 4 (right) which shows that after imposing the discretization of integral term, it would be dense. However, whatever the values are away from the diagonals their absolute values are getting smaller and smaller. This is another advantage of the new scheme at which one may obtain results of higher accuracies by solving sets of ODEs of reasonable sizes.

To check the stability of the computational procedure of WRBF, it is necessary to check that all the eigenvalues of the coefficient matrix $\tilde{\mathbf{B}}$ in (40) have negative real parts, in other words they locate in the left side of the complex plane. This is pursued numerically for different values of the number of spatial nodes in Figure 5. In light of the results, it can be inferred that all the eigenvalues have negative reals parts which is the beginning condition for the time-stepping solver to converge. Besides, by increasing the number of nodes, the largest eigenvalue (in absolute sign) gets bigger which put on show that the system is getting stiff and a time-stepping solver with a larger stability region or more number of temporal discretization nodes must be applied.

From computational results observed in Table 3 for the proposed WRBF scheme, it could be conjectured that there is a relation among the required number of time steps (in the forward Euler time-stepping scheme) and the number of spatial nodes. It could be written that the following relation could provide a (mild) stability condition 

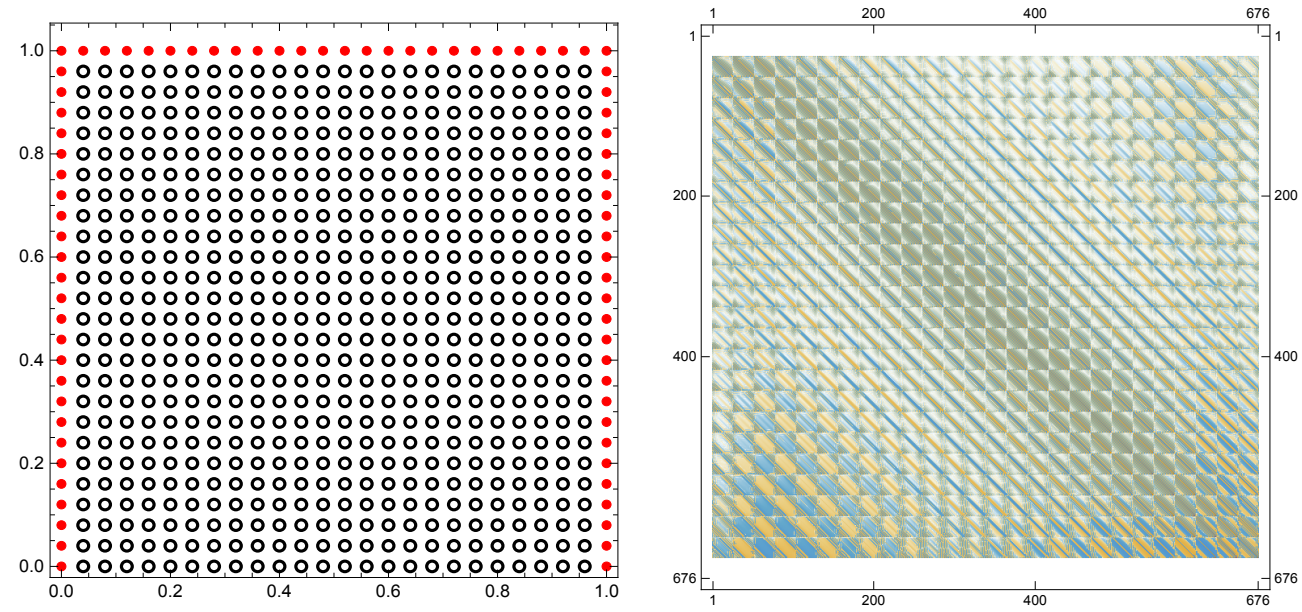

Figure 4: The computational grid along its boundaries (left) and the plot of the coefficient matrix $\tilde{\mathbf{B}}$ for $m=26, n=26$ for the WRBF method.

\begin{tabular}{|c|c|c|c|c|c|c|c|}
\hline Method & $m$ & $n$ & Size & $k$ & Price $U$ & Error & CPUTime \\
\hline FD & & & & & & & \\
& 16 & 16 & 256 & 201 & 6.51049 & $3.53201 \times 10^{-1}$ & 0.85 \\
& 30 & 25 & 725 & 501 & 5.97330 & $1.83987 \times 10^{-1}$ & 4.31 \\
& 40 & 40 & 1600 & 1001 & 6.05925 & $9.80335 \times 10^{-2}$ & 14.12 \\
& 60 & 50 & 3000 & 2001 & 6.10135 & $5.59411 \times 10^{-2}$ & 64.91 \\
& 70 & 70 & 4900 & 5001 & 6.11918 & $3.81049 \times 10^{-2}$ & 162.15 \\
\hline WRBF & & & & & & & \\
& 20 & 14 & 280 & 281 & 5.85113 & $3.06154 \times 10^{-1}$ & 5.85 \\
& 22 & 22 & 484 & 501 & 5.28339 & $8.73899 \times 10^{-1}$ & 19.95 \\
& 24 & 24 & 576 & 601 & 6.17289 & $1.55974 \times 10^{-2}$ & 30.69 \\
& 26 & 26 & 676 & 2001 & 6.16721 & $9.92461 \times 10^{-3}$ & 46.06 \\
\hline
\end{tabular}

Table 3: Results of comparisons for Test Problem 6.2.

for the numerical treatment discussed in this work, $k>m \times n$, or alternatively

$$
\frac{m n}{k}<1 .
$$

Figure 6 illustrates that how the numerical solution evolves once this condition is broken using the local WRBF method in Experiment 6.2.

From Table 3 we can state that for a fixed absolute error, e.g., $10^{-2}$, FD requires (around) $m=70, n=70$ points for spatial and $k=4901$ for temporal discretization, while for this accuracy we only need (around) $m=24, n=24$ and $k=601$, number points for discretizations, and thus $(70 \times 70) /(24 \times 24) \simeq 8$ times more spatial points and subsequently $162.15 / 46.06 \simeq 3$ more CPU times are required for FD in contrast to the WRBF.

It is remarked that the values obtained for the error reductions in Tables 1-4 are not necessarily uniform. A nonuniform convergence pattern is not at all surprising and it is also experienced by other authors dealing with the numerical approximation of the Bates model for American options (see, e.g, [7]). As a matter of fact, the solution being sought is affected by several kinds of irregularities (e.g., the Bates model is singular for $\nu=0$, [7, p. 1307]). Hence, the convergence of numerical discretization schemes is irregular as well.

Test Problem 6.3. [37] Using the same parameters as in Test Problem 6.2, here we study the variation of the resultant error for an American option under the Bates model.

The results based on the choice $\varrho=10$ as the penalty term (9) for this test are put together in Table 4 . The 

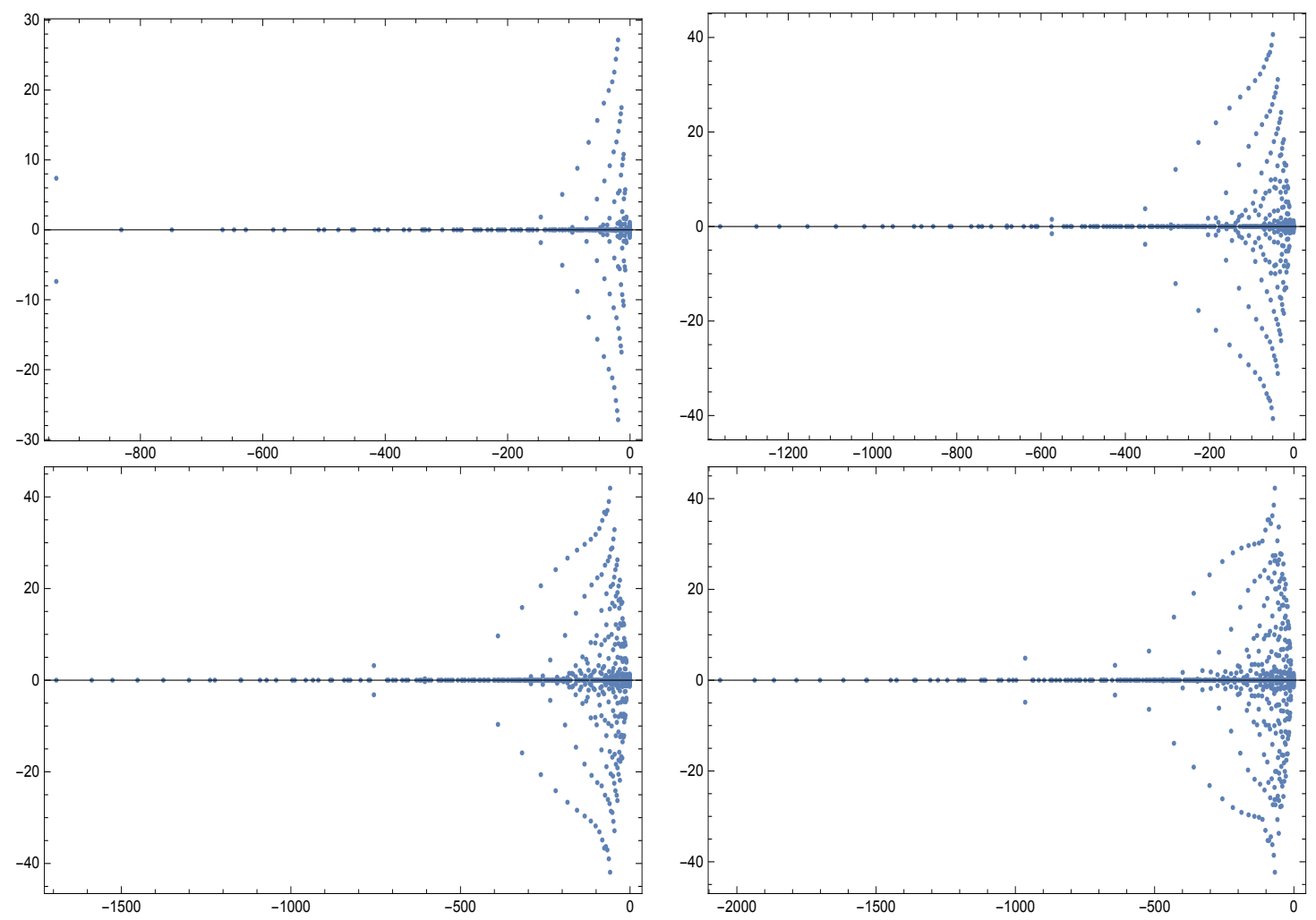

Figure 5: Distribution of the eigenvalues of the coefficient matrices $\tilde{\mathbf{B}}, m=20, n=14$ in top left, $m=22, n=22$ in top right, $m=24, n=24$ in bottom left, $m=26, n=26$ in bottom right.

computational domain for the FD is $\Omega=[0,4 E] \times[0,1]$ (exactly based on [37]) and the transformation of $\Omega=$ $[0,3 E] \times[0,1]$ to $\Omega=[0,1] \times[0,1]$ for the WRBF.

The explicit mid-point time-stepping scheme which possess a higher rate of convergence and higher stability region is used for both the FD and the WRBF schemes in Table 4 in order to handle the American options (which are nonlinear after semi-discretization process) as quickly and efficiently as possible.

Here, in the practical implementations of the American options, we consider $U\left(S_{\max }, \nu, \tau\right) \approx S_{\max }-E$ for the boundary at $S \rightarrow+\infty$. Due to this, the vector $b(\tau)$ in (40) is zero.

For this problem, we used the idea of trial-and-error [19, section 17.1.1] for finding a good shape parameter (not an optimal one). Hence, we have a different $c$ corresponding to each number of spatial nodes.

Once again, the option price can be computed with a high level of accuracy in a short piece of time using the WRBF while FD requires much more spatial nodes in order to reach higher accuracies. Since our main aim is to price stochastic volatility jump models via moderate-size semi-discrete systems in a small piece of time via the local Wendland RBF meshfree method, we do not consider more spatial nodes to get prices of higher accuracies (more than $10^{-3}$ ). Similar to (65) and the theoretical stability bound (59), we may write a numerical stability bound for the number of temporal nodes in contrast to the number of spatial nodes for the explicit mid-point method as $\frac{m n}{k}<1$.

Numerical results shown in Table 4 indicate that the new WRBF solution provides an accurate solution of the pricing American options in contrast to the standard procedures, such as FD. The results agreed with the results of the AFD scheme given in Table 2. Furthermore, it is necessary to check the numerical rate of convergence for the explicit mid-point method (45) especially in the American option pricing case. Due to this, in Table 5, the stability and numerical convergence order of (45) is getting checked out by halving the temporal step size for a fixed spatial 

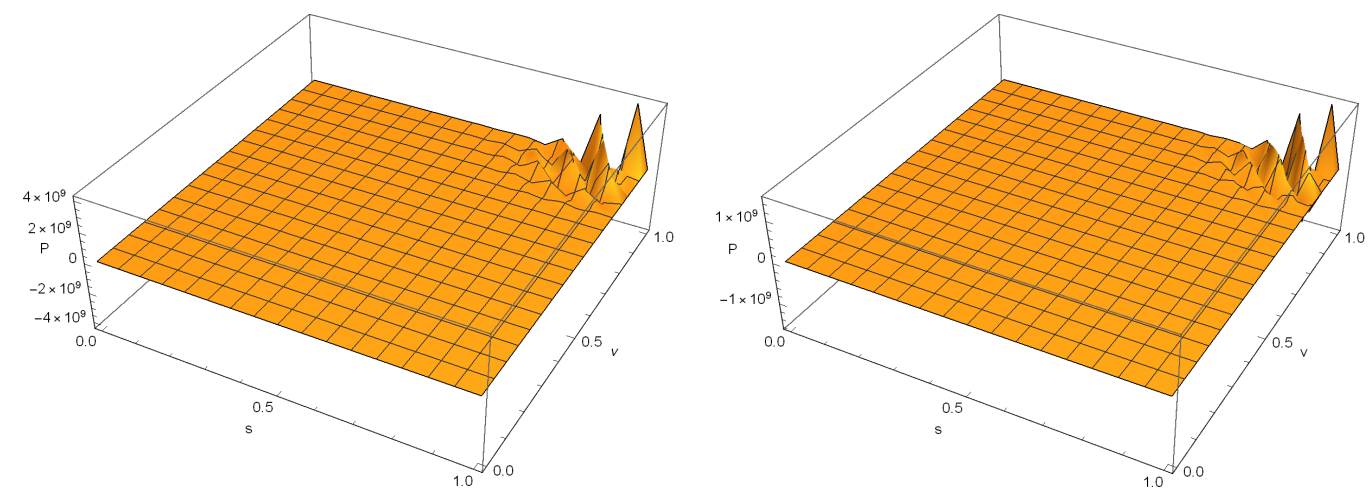

Figure 6: The numerical solution with the broken stability condition (65) in Test Problem 6.2, when $m=n=22$ and $k=325<22 \times 22$ in left and $m=n=26$ and $k=501<26 \times 26$ in right.

\begin{tabular}{|c|c|c|c|c|c|c|c|}
\hline Method & $m$ & $n$ & Size & $k$ & Price $U$ & Error & CPUTime \\
\hline FD & & & & & & & \\
& 16 & 16 & 256 & 101 & 6.51397 & $3.52860 \times 10^{-1}$ & 12.17 \\
& 22 & 22 & 484 & 251 & 6.01610 & $1.45012 \times 10^{-1}$ & 19.45 \\
& 32 & 32 & 1024 & 401 & 6.04772 & $1.13385 \times 10^{-1}$ & 89.65 \\
& 42 & 42 & 1764 & 1001 & 6.05446 & $1.06644 \times 10^{-1}$ & 474.72 \\
& 52 & 52 & 2704 & 1251 & 6.08157 & $7.95339 \times 10^{-2}$ & 799.75 \\
\hline WRBF & & & & & & & \\
$c=43$ & 18 & 16 & 288 & 201 & 6.15899 & $2.11433 \times 10^{-3}$ & 12.95 \\
$c=3$ & 20 & 20 & 400 & 401 & 6.16658 & $5.47023 \times 10^{-3}$ & 35.75 \\
$c=60$ & 23 & 18 & 414 & 401 & 6.16346 & $2.35619 \times 10^{-3}$ & 41.67 \\
$c=59$ & 26 & 26 & 676 & 651 & 6.17034 & $9.23562 \times 10^{-3}$ & 124.30 \\
\hline
\end{tabular}

Table 4: Comparison of convergence history for Test Problem 6.3.

discretization $(m=20, n=20, c=3)$ via the following relation:

$$
\mathrm{ROC} \approx\left|\log _{2} \frac{P_{\text {approx }}\left(\Delta_{\tau}\right)-P_{\text {approx }}\left(\Delta_{\tau} / 2\right)}{P_{\text {approx }}\left(\Delta_{\tau} / 2\right)-P_{\text {approx }}\left(\Delta_{\tau} / 4\right)}\right| .
$$

The results in Table 5 confirm the stability bound (65) as well as a second order of convergence along the temporal variables, which is quite fast with better stability region in contrast to the first order Euler's method (42) that we applied for the European case (linear PIDEs).

We too stress that the condition number of the collocation matrix (in the case of FD or global meshfree methods) is very sensitive to selection of the shape parameter $c$ [22], the numbers of supporting nodes $N=m \times n$ and the system gets ill-conditioned instantly. But in our case, this dependence is low.

\section{Summary}

In this paper, a local RBF method for pricing European and American options on an underlying asset described by the Bates model was proposed. It was discussed how the Wendland compactly supported radial function along a good shape parameter allow us to obtain a numerical method for this kind of problem. In all the numerical simulations performed, absolute errors are of accuracy order $10^{-3}$.

Moreover, the discretization of the non-local integral operator was performed efficiently, as it requires about the same computer time as is necessary to discretize (all) the other local operators in the PIDE. It is also worth noticing that good results are also obtained when the variance process is extremely close the origin, or when the option's maturity is long. 


\begin{tabular}{|c|c|c|}
\hline$k$ & Price $U$ & ROC \\
\hline 201 & Fail & - \\
401 & 6.1665782270 & - \\
801 & 6.1665818490 & - \\
1601 & 6.1665827713 & 1.97 \\
3201 & 6.1665830020 & 2.00 \\
\hline
\end{tabular}

Table 5: Checking the stability and convergence rate along time for Test Problem 6.3.

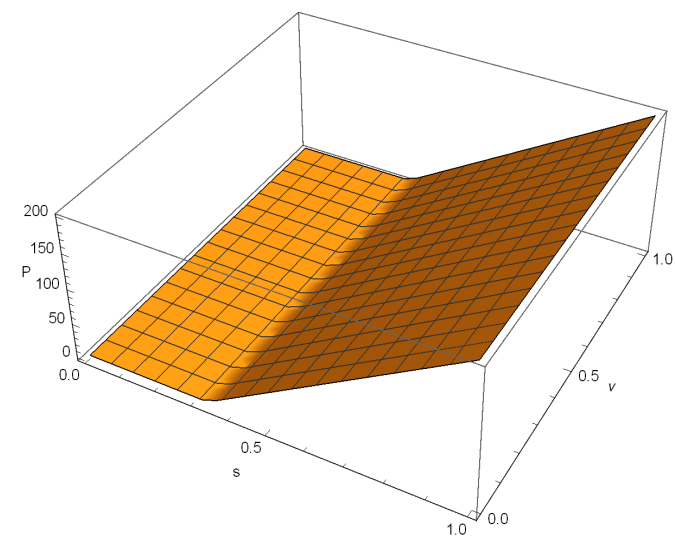

(a) $\tau=0$

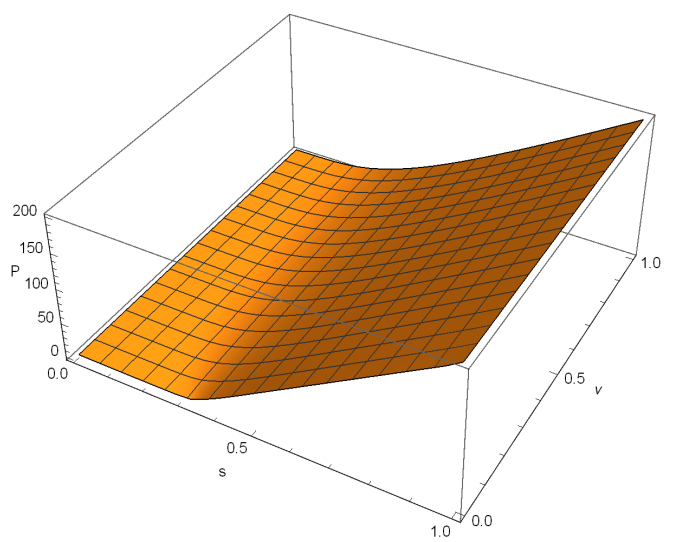

(b) $\tau=0.5$

Figure 7: The numerical solution of local WRBF for American option under the Bates model in Experiment 6.3.

\section{Acknowledgements}

This work has partially been supported by the Ministerio de Ciencia, Innovación y Universidades Spanish grant MTM2017-89664-P. The authors are grateful to a number of corrections and comments made by two referees which helped improve this paper.

\section{References}

[1] M. Aichinger, A. Binder, A Workout in Computational Finance, Wiley, UK, 2013.

[2] J. Amani Rad, K. Parand, Pricing American options under jump-diffusion models using local weak form meshless techniques, Int. J. Comput. Math., 94 (2017), 1694-1718.

[3] L. Andersen, J. Andreasen, Jump-diffusion processes: volatility smile fitting and numerical methods for pricing, Rev. Derivatives Res. 4 (2000), 231-262.

[4] T. Apel, G. Winkler, U. Wystup, Valuation of options in Heston's stochastic volatility model using finite element methods, J. Hakala, U. Wystup, eds. Foreign Exchange Risk: Models, Instruments and Strategies. Risk Books, London, UK, 2002.

[5] M.L. Abell, J.P. Braselton, Mathematica by Example, Fifth Edition, Academic Press, 2017.

[6] L.V. Ballestra, C. Sgarra, The evaluation of American options in a stochastic volatility model with jumps: An efficient finite element approach, Comput. Math. Appl., 60 (2010), 1571-1590.

[7] L.V. Ballestra, L. Cecere, A fast numerical method to price American options under the Bates model, Comput. Math. Appl., 72 (2016) $1305-1319$

[8] O.E. Barndorff-Nielsen, N. Shephard, Ornstein-Uhlenbeck-based models and some of their uses in financial economics (with discussion), J. Royal Stat. Soc. B, 63 (2001), 167-241.

[9] D. Bates, Jumps and stochastic volatility: the exchange rate processes implicit in Deutsche mark opions, Rev. Fin. Studies, 9 (1996), 69-107.

[10] D.S. Bates, Bates Model in: R. Cont (ed.): Encyclopedia of Quantitative Finance, Wiley, USA, 2010. 
[11] R.T.L. Chan, S. Hubbert, A numerical study of radial basis function based methods for options pricing under the one dimension jumpdiffusion model, Tech. Rep., arXiv 1011.5650v4, (2011), 27 pages.

[12] C. Chiarella, B. Kang, G.H. Meyer, A. Ziogas, The evaluation of American option prices under stochastic volatility and jump-diffusion dynamics using the method of lines, Int. J. Theor. Appl. Finance, 12 (2009), 393-425.

[13] R. Company, V. Egorova, L. Jódar, C. Vázquez, Finite difference methods for pricing American put option with rationality parameter: Numerical analysis and computing, J. Comput. Appl. Math., 304 (2016), 1-17.

[14] R. Cont, P. Tankov, Financial Modelling with Jump Processes, Chapman \& Hall/CRC Financial Mathematics Series, UK, 2004.

[15] R. Cont, E. Voltchkova, Finite difference methods for option pricing in jump-diffusion and exponential Lévy models, SIAM J. Numer. Anal., 43 (2005), 1596-1626.

[16] S. Cox, P. Matthews, Exponential time differencing for stiff systems, J. Comput. Phys., 176 (2002), 430-455.

[17] B. Düring, M. Fournié, High-order compact finite difference scheme for option pricing in stochastic volatility models, J. Comput. Appl. Math. 236, (2012), 4462-4473.

[18] M. Fakharany, R. Company, L. Jódar, Positive finite difference schemes for a partial integro-differential option pricing model, Appl. Math. Comput., 249 (2014), 320-332.

[19] G. Fasshauer, Meshfree Approximation Methods with MATLAB, World Scientific Publishing Co., Singapore, 2007.

[20] G.E. Fasshauer, Solving partial differential equations by collocation with radial basis functions, in: A.L. Me'chaute' (Ed.), Proceedings of Chamonix, Vanderbilt University Press, Nashville, TN, 1996, pp. 1-8.

[21] P.A. Forsyth, K.R. Vetzal, Quadratic convergence for valuing American options using a penalty method, SIAM J. Scient. Comput., 23 (2002), 2096-2123.

[22] P.G. Giribone, S. Ligato, Option pricing via radial basis functions: Performance comparison with traditional numerical integration scheme and parameters choice for a reliable pricing, Int. J. Financial Eng., 2 (2015), 30 pages.

[23] R. Hardy, Multiquadric equations of topography and other irregular surfaces, J. Geophys. Res., 176 (1971), 1905-1915.

[24] S. L. Heston, A closed-form solution for options with stochastic volatility with applications to bond and currency options, Rev. Fin. Stud., 6 (1993), 327-343.

[25] K.J.I. Hout, S. Foulon, ADI finite difference schemes for option pricing in the Heston model with correlation, Int. J. Numer. Anal. Modeling, 7 (2010), 303-320.

[26] A. Jacquier, M. Keller-Ressel and A. Mijatović, Large deviations and stochastic volatility with jumps: asymptotic implied volatility for affine models, Stochastics, 85 (2013), 321-345.

[27] M. Kelly, Evaluation of financial options using radial basis functions in Mathematica, The Mathematica J., 11 (2009), $333-357$.

[28] A. Lipton, The vol smile problem, Risk, February (2002), 81-85.

[29] A. Melino, S.M. Turnbull, Pricing foreign currency options with stochastic volatility, J. Econometrics, 45 (1990), $239-265$.

[30] R. Merton, Option pricing when underlying stock returns are discontinuous, J. Fin. Ecom., 3 (1976), 125-144.

[31] O. Pironneau, F. Hecht, Mesh adaptation for the Black \& Scholes equations, East-West J. Numer. Math., 8 (2000), $25-35$.

[32] S. Salmi, J. Toivanen, An iterative method for pricing American options under jump-diffusion models, Appl. Numer. Math., 61 (2011), 821-831.

[33] L.O. Scott, Pricing stock options in a jump-diffusion model with stochastic volatility and interest rates: applications of Fourier inversion methods, Math. Finance, 7 (1997), 413-426.

[34] E. Süli, D. Mayers, An Introduction to Numerical Analysis, Cambridge University Press, UK, 2003.

[35] D. Tavella, C. Randall, Pricing Financial Instruments — The Finite Difference Method, John Wiley \& Sons, Chichester, 2000.

[36] J. Toivanen, A componentwise splitting method for pricing American options under the Bates model, Applied and Numerical Partial Differential Equations: Scientific Computing, Simulation, Optimization and Control in a Multidisciplinary Context, in: Computational Methods in Applied Sciences, vol. 15, Springer, (2010), 213-227.

[37] L. von Sydow, J. Toivanen, C. Zhang, Adaptive finite differences and IMEX time-stepping to price options under Bates model, Int. J. Comput. Math., 92 (2015), 2515-2529.

[38] H. Wendland, Piecewise polynomial, positive definite and compactly supported radial functions of minimal degree, Adv. Comput. Math., 4 (1995), 389-396. 